\title{
Spatial Mode Demultiplexer Based on Spatial Phase Filters for Optical Communication
}

\author{
Yu Zhang ${ }^{1, a}$, LiMing Tian ${ }^{2, b}$, Jian $\mathrm{Qu}^{2, \mathrm{c}}$ \\ ${ }^{1}$ National Ocean Technology Center, Tianjin, 300112, China \\ ${ }^{2}$ Information Center, State Administration for Quality Supervision and Inspection and Quarantine, \\ Beijing, 100088, China \\ azhyunotc@sina.com, btianlmsaqs@sina.com, 'cqujiansaqs@sina.com
}

Keywords: Mode division multiplexing, optical communication, mode demultiplexer, phase filter.

\begin{abstract}
In this paper, a spatial mode demultiplexer is proposed to recover modes which are weakly coupled in mode division multiplexed optical fiber communication systems. A spatial light modulator is employed to modulate the spatial phase as the kernel of the DEMUX. In the simulation, we explore the position of the observed plane, the phase patterns of the spatial phase filter and the group of the modes, which will exercise a great influence on the mode demultiplexing.
\end{abstract}

\section{Introduction}

The network traffic created, replicated, and transported by individuals and machines, such as cloud-based services, has increased rapidly with growth rates about $50 \%$ per year [1]. So galloping information is creating a strain on the optical fiber communication, which is the backbone for the telecommunications infrastructure [2]. This trend is expected to continue for the foreseeable future, and the single mode fibers (SMFs) are rapidly approaching the Shannon capacity limit. Mode division multiplexing (MDM), with data multiplexed over orthogonal spatial channels of the eigenmodes in the few mode fibers (FMFs) or the multimode fibers (MMFs), has attracted much attention as a potential approach against the capacity crunch of SMFs [1-3].

The development of specific components are required to realize the full mode multiplexed transmission system with FMFs or MMFs. The spatial mode demultiplexer (DEMUX) is one of the key elements in mode division multiplexing. Phase plates are used to convert the multiplexed higher order modes to single mode LP01 as a conventional solution [4]. Photonic lanterns, where the FMF is tapered into $\mathrm{N}$ output SMFs, are the other technique to build a mode DEMUX [5]. However, these two types of DEMUX will lead to strong crosstalk between the multiplexed modes, and signal processing at the receiver end is required to demultiplex modes.

In this paper, we propose a spatial mode demultiplexer based on spatial phase filters for MDM optical fiber communication systems. This kind of spatial phase filters employs a spatial light modulator (SLM) as the kernel of the DEMUX. First, we establish a simulated system for mode demultiplexing. Then we compare the demultiplexed results on two different positions of the observed plane. Finally, we explore the phase patterns of the spatial phase filter and the group of the modes, which will exercise a great influence on the mode demultiplexing.

\section{Theory of the spatial modes demultiplexer}

The setup of the spatial modes demultiplexer is shown in Fig.1. The light from the FMF, in which multi-modes are multiplexed together, is collimated through the fiber collimator. After through a polarizer, this collimated incident beam is modulated by the SLM which is implemented as a spatial phase filter. Then after transmitting through a lens, the modulated light can be detected by the CCD, which is employed to detect the quality of the demultiplexed modes. 


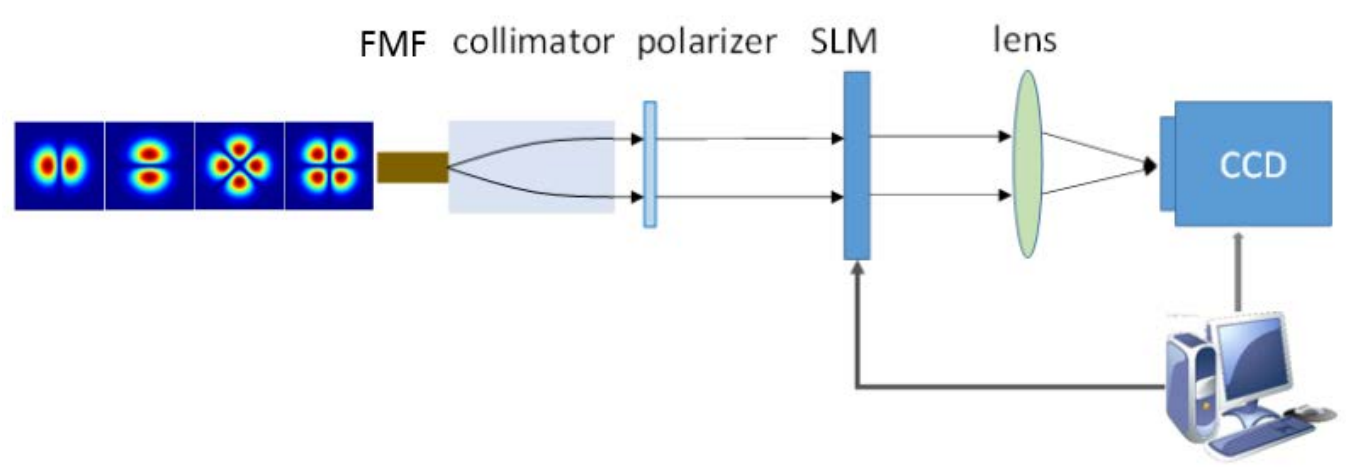

Fig.1 The setup of the spatial modes demultiplexer. FMF: few mode fiber; SLM: spatial light modulator.

\section{The effect of the position of the observed plane}

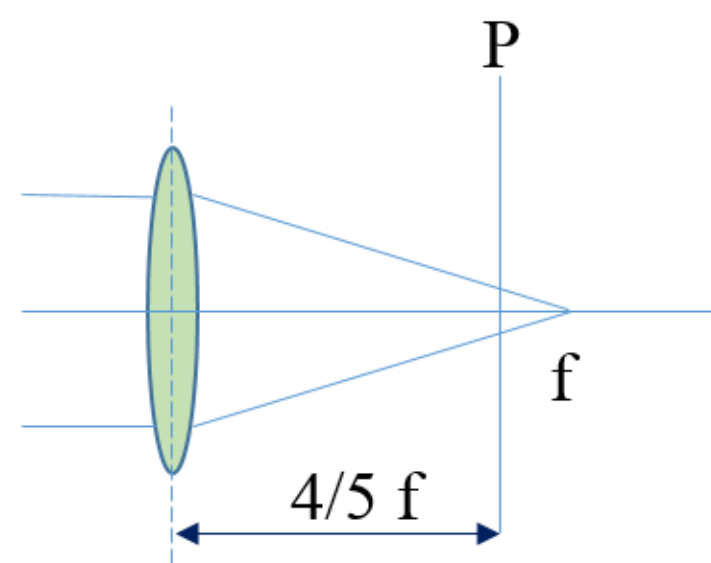

(a)

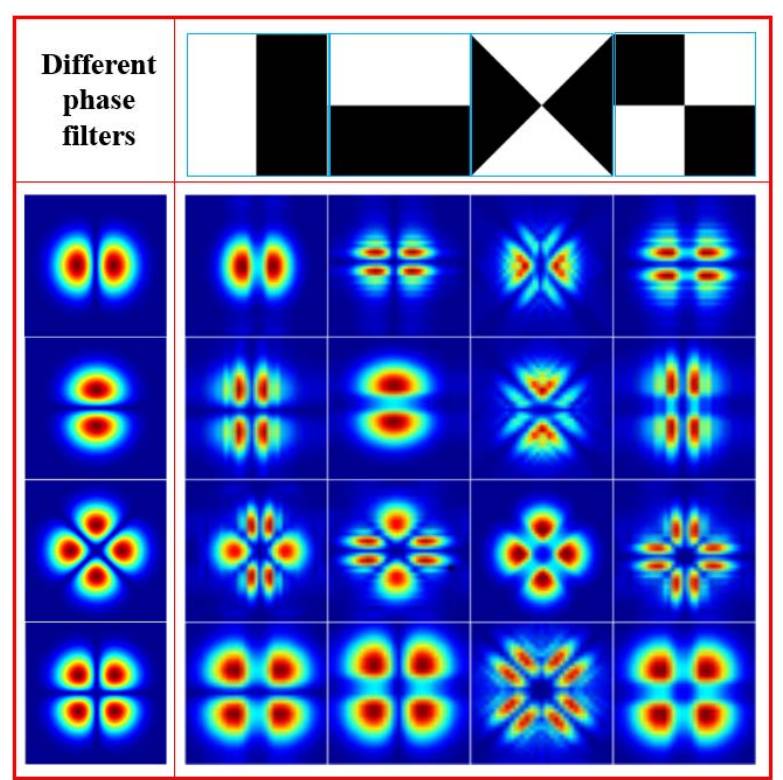

(b)

Fig. 2 The simulated results on the plane $1 / 5$ focus before the focus plane. (a) The observed plane 1/5 focus before the focus plane. (b) The LP11a, LP11b, LP21a and LP21b modes are modulated by four different phase filters, respectively.

The simulated results on the plane 1/5 focus from the focus plane are shown in Fig. 2. In Fig. 2(a), the CCD is placed on the plane 4/5 focus behind the lens. Fig.2 (b) illustrates the observed results of several modes modulated by four different phase filters, respectively. The first row is the phase filters, and they are LP11a-phase, LP11b-phase, LP21a-phase and LP21b-phase from left to right. The first column is the input modes, and they are LP11a, LP11b, LP21a and LP21b from top to bottom. The patterns of the diagonal line are the results of the input modes modulated by their corresponding spatial phase filters. However, the input modes are not converted to the fundamental mode LP01, whose power at the center is the highest.

In contrast, the simulated results on the focus plane have been converted to the fundamental mode LP01 on the diagonal line in Fig. 3 (b). Fig.3 illustrates the simulated results on the focus plane. The observed plane is just the focus plane in Fig.3 (a). The LP11a, LP11b, LP21a and LP21b modes are modulated by four different phase filters in Fig.3 (b), respectively. In Fig.3 (b), the patterns of the diagonal line are the results of the input modes modulated by their corresponding spatial phase filters. And we can find that the input modes are all converted to the fundamental mode LP01. 

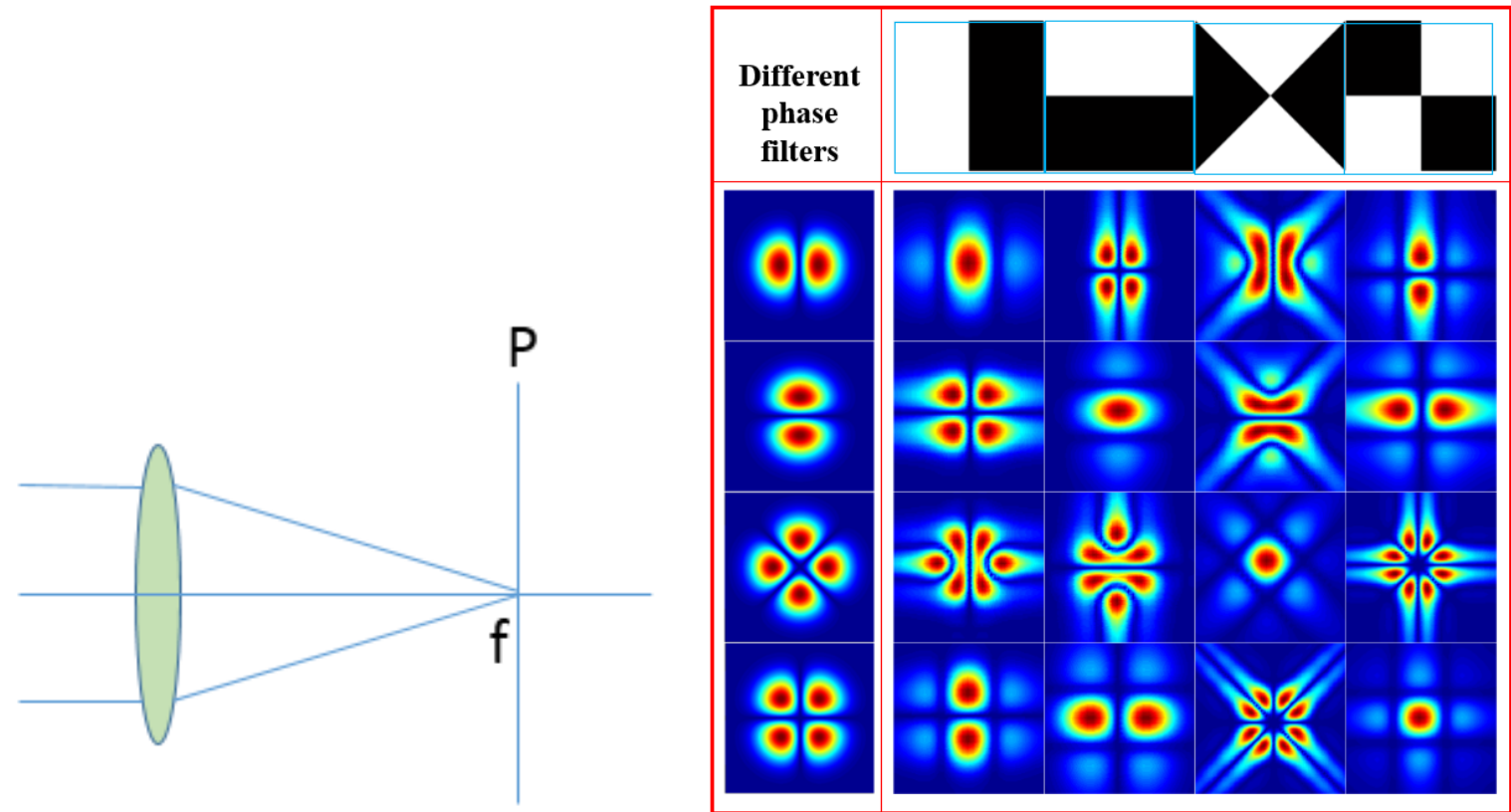

Fig.3 The simulated results on the focus plane. (a) The observed plane is just the focus plane. (b) The LP11a, LP11b, LP21a and LP21b modes are modulated by four different phase filters, respectively.

Input multi-modes demultiplexed with different phase filters

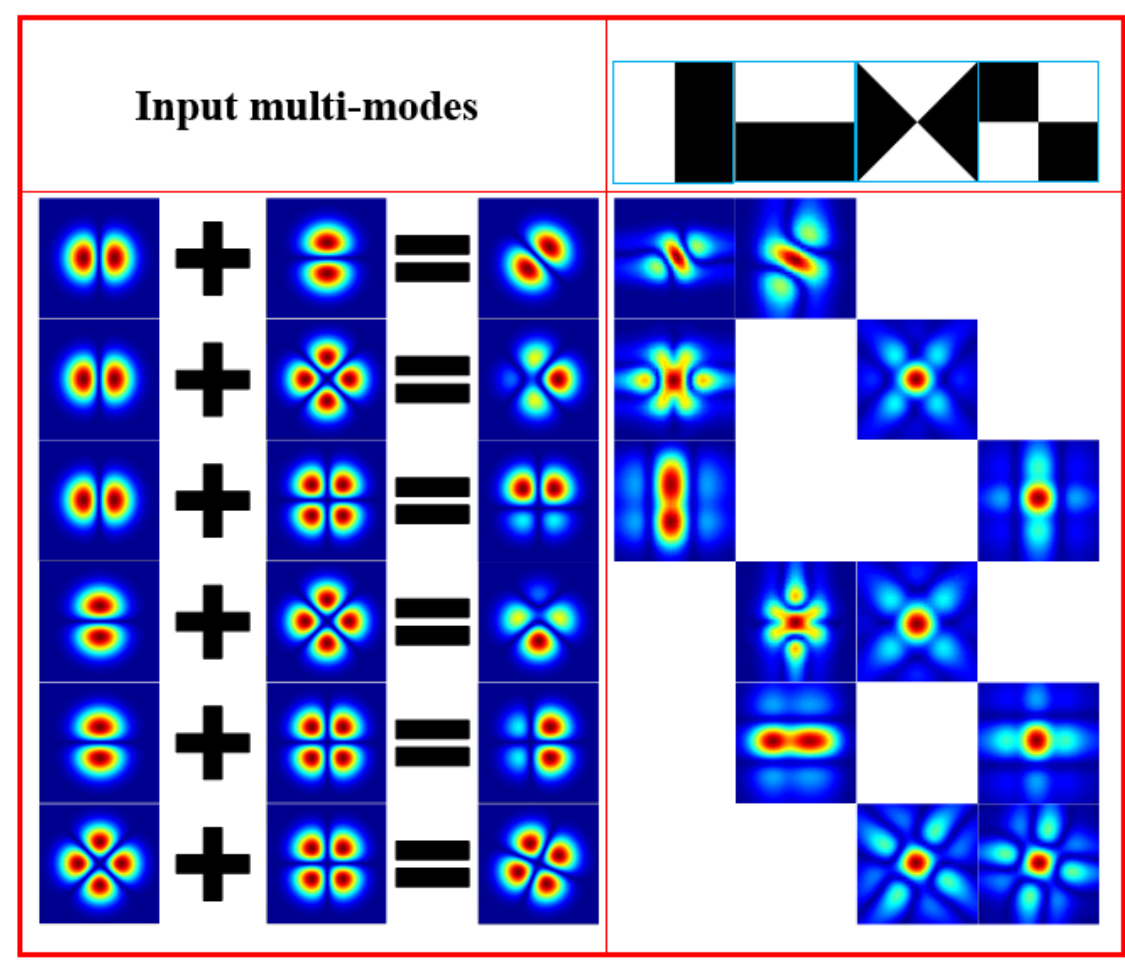

Fig.4 The observed patterns of input multi-modes demultiplexed by different spatial phase filters.

Fig. 4 illustates the observed patterns of input multi-modes demultiplexed by different spatial phase filters. The first row is the phase filters, and they are LP11a-phase, LP11b-phase, LP21a-phase and LP21b-phase from left to right. The first column is the input multi-modes, and they are LP11a+LP11b, LP11a+LP21a, LP11a+LP21b, LP11b+LP21a, LP11b+LP21b and LP11a+LP21b from top to bottom. The patterns of the diagonal line are the results of the input multi-modes demultiplexed by their corresponding spatial phase filters. 


\section{Summary}

In this paper, we proposed a spatial mode DEMUX to recover modes in MDM optical fiber communication systems. We employed a spatial light modulator to as a spatial phase filter which is the kernel of the DEMUX. In the simulation, we explore the position of the observed plane, the phase patterns of the spatial phase filter and the group of the modes, which will exercise a great influence on the mode demultiplexing.

\section{References}

[1]. D. Richardson, J. Fini, and L. Nelson: Space-division multiplexing in optical fibres, Nature Photonics 7, 354-362 (2013).

[2]. P. J. Winzer: Making spatial multiplexing a reality, Nat Photon, 345-348(2014).

[3]. R. J. Essiambre, R. Ryf, N. K. Fontaine, and S. Randel: Breakthroughs in Photonics 2012: Space-Division Multiplexing in Multimode and Multicore Fibers for High-Capacity Optical Communication, Ieee Photonics J 5(2013).

[4]. Montero C, Moreno V, Prieto-Blanco X, et al: Fabrication and characterization of ion-exchanged glass binary phase plates for mode-division multiplexing, Proc Spie, (2012)

[5]. S. G. Leon-Saval, A. Argyros, and J. Bland-Hawthorn, "Photonic lanterns," Nanophotonics 2, 429-440 (2013). 